\title{
Texture Enhancement using Diffusion Process with Potential
}

\author{
Emmanuel Cohen \\ Department of Electrical Engineering \\ Technion - Israel Institute of Technology \\ Technion City, Haifa 32000, Israel, and \\ CEREMADE, UMR 7534 CNRS \\ Université Paris Dauphine, PSL*, France
}

\author{
Laurent D. Cohen \\ CEREMADE, UMR 7534 CNRS \\ Université Paris Dauphine \\ PSL $^{*}$, France
}

\author{
Yehoshua Y. Zeevi \\ Department of Electrical Engineering \\ Technion - Israel Institute of Technology \\ Technion City, Haifa 32000, Israel
}

\begin{abstract}
Applications of anisotropic diffusion equation to texture enhancement have shown that an image can be smoothed while preserving high frequency features like edges. However, preserving textures still remains challenging. One way to preserve textures is by adding an extra term to the diffusion equation. This additional term can be interpreted as a potential, similar to the role of Schrödinger's potential in the complex diffusion equation, or as a reaction term in a reaction-diffusion process. We show the effect of such potentials on texture denoising, highlighting that anisotropic diffusion with potential combines properties of diffusion (piecewise-smoothing) and potential (enhancing fine structures) filters. Simulations performed on pure texture samples indicate that the reconstruction depends on the type of texture and the transform operator used in the potential. The diffusionwith-potential approach is extended. Local and nonlocal results show that nonlocal diffusion improves the quality of denoising.
\end{abstract}

\section{INTRODUCTION}

Applying the linear diffusion equation to an image is equivalent to convolving it with a Gaussian filter. Its lowpass properties enable image smoothing, removing noise but, also, degrading high-frequency features (like edges) [1]. To overcome this problem, Perona and Malik (PM) [2] introduced a nonlinear diffusion process, where an anisotropic diffusion coefficient slows the diffusion near edges. Diffusivity is generally selected to be a decreasing function of the gradient norm of the image. The minimization of the total variation functional led to the TV restoration model, based on a convex diffusivity function [3]. Other models for diffusivity have been suggested to improve features preservation (e.g. [4]). Forward-and-Backward (FAB) diffusion filtering even enhances image features such as edges and some types of textures [5]. Other improvements of the nonlinear diffusion equation, e.g. regularized or tensor-diffusivity models, further enhance the quality of PDE-based denoising processes [1]. Diffusion equation was originally applied in the real domain but a complex version has been proposed by Gilboa et al. [6]. The use of a complex diffusion, inspired by the Schrödinger equation, has highlighted a new aspect of diffusion processes: both edge detection (highpass) and denoising (lowpass) can be achieved at the same time [6]. Compared with the classical anisotropic diffusion, the complex diffusion achieves almost the same denoising in fewer iterations.

The PM-type diffusion produces piecewise-smooth filtered images [7], however, small repeated patterns like textures are compromised or lost. Texture preservation calls for more robust methods. Similarly to the way a particle can be trapped in a physical potential, Honigman and Zeevi [8] have shown how the addition of a potential to the diffusion equation can enhance textured images. To this end they used wavelet shrinkage. Plonka and Ma [9] used wave atom shrinkage.

We study such potentials and their impact on different types of textures. Assuming that images are composed of differentlytextured patches, we are looking for the appropriate potentials. We extend the study to the nonlocal approach introduced by G. Gilboa and Osher [10], and compare between local and nonlocal denoising methods.

\section{LOCAL DIFFUSION PROCESSES}

Let $\tilde{u}_{0}: \Omega \subset \mathbb{R}^{2} \rightarrow[0,1]$ be the original image of size $\sqrt{N} \times \sqrt{N}$, where $N=2^{l}$ and $l$ is an even number. The signal is contaminated with additive white Gaussian noise $n$. The noisy image $u_{0}(x)$ at pixel $x$ is given by:

$$
u_{0}(x)=\tilde{u}_{0}(x)+n(x) .
$$

Solving the linear diffusion equation,

$$
\left\{\begin{array}{l}
\partial_{t} u=\Delta u \\
u(x, 0)=u_{0}(x)
\end{array}\right.
$$

consists of convolving the input image $u_{0}$ with smoothing kernels which approximate Gaussian kernel functions of standard deviations $\mu_{t}=\sqrt{2 t}$. This diffusion process illustrates the scale-space theory [1]. However, it presents several limitations. Despite good smoothing properties, many details are lost.

To cope with this problem, the adaptive anisotropic process of PM [2] allows preferential diffusion as follows:

$$
\partial_{t} u=\operatorname{div}(g(|\nabla u|) \nabla u),
$$

where $|\nabla u|=\sqrt{u_{x}^{2}+u_{y}^{2}}$ is the Euclidian norm of the gradient 
of $u$, and the diffusivity $g: \mathbb{R} \rightarrow \mathbb{R}^{+}$is a non-negative decreasing function satisfying $\lim _{r \rightarrow \infty} g(r)=0$. Thus, only pixels with relatively low gradient intensities (corresponding to high values of $g$ ) are affected and the edges, where the norm of the gradient is typically high, are preserved. Several models for $g$ have already been proposed (e.g. [2], [4], [11]). We consider the PM model, for $r \in \mathbb{R}$ :

$$
g(r)=\frac{1}{1+\frac{r^{2}}{k^{2}}},
$$

where $k \in \mathbb{R}^{*}$ is a constant parameter characterizing the gradient intensity below which the pixels are highly diffused. The resulting filtered images by anisotropic diffusion are piecewise-smooth and edges are preserved.

Catté et al. [12] proposed a new model that should avoid the theoretical problem aroused by anisotropic diffusion, i.e. ill-posedness of the equation despite the existence of discrete solutions, known as the Perona-Malik paradox [1]. They replaced the diffusivity $g(|\nabla u|)$ by $g(|\nabla(G * u)|)$, where $G$ is a Gaussian kernel and $*$ the convolution operator, as follows:

$$
\partial_{t} u=\operatorname{div}(g(|\nabla(G * u)|) \nabla u) .
$$

Ratner and Zeevi resolved the PM paradox by showing that discrete approximation of the diffusion equation is equivalent in fact to the Telegraph-Diffusion equation [13]. Also, smoothing the noisy image before computing the diffusivity allows removing part of the noise and avoids staircasing [1]. The regularized anisotropic diffusion (RAD) equation (4) is thus more appropriate to distinguish between noise (to remove) and edges (to preserve).

Various structured textures can be considered to be highfrequency oscillations. Diffusion-based filtering often removes them after few iterations. To counter this effect, one may add some features containing textural information to a potential [8]. Equation (4) is then modified as follows:

$$
\partial_{t} u=\operatorname{div}(g(|\nabla(G * u)|) \nabla u)+\alpha V u_{0},
$$

where $V u_{0}$ is some kind of transformation of the input image $u_{0}$, called potential [8] or reaction term [9], and $\alpha$ is a constant parameter which controls the influence of the potential on the denoising process. Since the potential should contain the vanishing (diffused) details, $V$ should be a highpass filter. A lowpass preprocessing cleans the input image while preserving fine structures, that are then extracted by subtracting $u_{0}$, as follows [9]:

$$
V=W^{-1} T_{\theta} W-I,
$$

Here, $T_{\theta}$ is typically a soft thresholding operator with threshold $\theta$, and $I$ is the identity operator. $W$ is a $2 \mathrm{D}$ transform operator and $W^{-1}$ is its inverse. Several authors (e.g. [8], [9]) have proposed to use wavelet shrinkage or wave atom shrinkage (WS and WAS respectively). The ultimate goal remains to synthesize a potential that will best extract the missing details, by judiciously choosing $W$. On natural textured images, such potentials usually improve denoising results. However, their impact on pure textures has not been clearly explored.

\section{NONLOCAL DIFFUSION PROCESSES}

PDEs extension to the nonlocal framework is based on a new definition of derivatives [10]. The nonlocal partial derivative of $u$ at point $x \in \Omega \subset \mathbb{R}^{2}$ with respect to point $y \in \Omega$ is:

$$
\partial_{y} u(x)=\frac{u(y)-u(x)}{d(x, y)},
$$

where $d$ is a positive measure defined on $\Omega \times \Omega$, that is supposed to be symmetric i.e. $d(x, y)=d(y, x)$ for all $x, y \in \Omega$, called distance. This definition is consistent with the general definition of the derivative operator in $\mathbb{R}^{2}$. It was inspired by the graph theory [14]. In fact, an image can be considered as a graph of pixels. Each pixel-vertex $x$ is connected to another $y$ with weight:

$$
w(x, y)=\frac{1}{d^{2}(x, y)} .
$$

The nonlocal gradient $\nabla_{\mathrm{NL}} u: \Omega \rightarrow \mathbb{R}^{N}$ is then defined as the vector of all partial derivatives:

$$
\nabla_{\mathrm{NL}} u(x)=((u(y)-u(x)) \sqrt{w(x, y)})_{y \in \Omega} .
$$

The nonlocal divergence of a vector $\vec{v}=(v(x, y))_{y \in \Omega}$ is given by:

$$
\operatorname{div}_{\mathrm{NL}} \vec{v}(x)=\int_{\Omega}(v(x, y)-v(y, x)) \sqrt{w(x, y)} d y .
$$

The above framework allows us to express diffusion processes in their nonlocal forms. The nonlocal linear diffusion equation is given by:

$$
\partial_{t} u=\operatorname{div}_{\mathrm{NL}}\left(\nabla_{\mathrm{NL}} u\right) .
$$

Using (9) and (10), it can be also written as:

$$
\partial_{t} u=2 \int_{\Omega}(u(y)-u(x)) w(x, y) d y .
$$

The steady state of the above equation corresponds to the Nonlocal Means algorithm (NLM) [15]:

$$
u(x)=\frac{\int_{\Omega} u(y) w(x, y) d y}{\int_{\Omega} w(x, y) d y} .
$$

Nonlocal linear diffusion is in this way a generalization of NLM. In [16], it is shown how nonlocal processes perform better results than local ones, and how an iterative procedure based on the steepest descent (11) outperforms the original nonlocal means algorithm, also in terms of computational complexity.

Similarly to the local method proposed by [2], we use a nonlocal version of anisotropic diffusion (NLAD):

$$
\partial_{t} u=\operatorname{div}_{\mathrm{NL}}\left(g\left(\left|\nabla_{\mathrm{NL}} u\right|\right) \nabla_{\mathrm{NL}} u\right) .
$$


Using (10), the last equation can be written as:

$$
\begin{aligned}
\partial_{t} u= & \int_{\Omega}(u(y)-u(x))\left(\frac{1}{1+\left|\nabla_{\mathrm{NL}} u\right|^{2}(x) / k^{2}}+\right. \\
& \left.\frac{1}{1+\left|\nabla_{\mathrm{NL}} u\right|^{2}(y) / k^{2}}\right) w(x, y) d y
\end{aligned}
$$

where the nonlocal norm of the gradient is defined by:

$$
\left|\nabla_{\mathrm{NL}} u\right|(x)=\sqrt{\int_{\Omega}(u(y)-u(x))^{2} w(x, y) d y} .
$$

Similarly to local diffusion with potential, we can compute nonlocal diffusion with potential, as follows:

$$
\partial_{t} u=\operatorname{div}_{\mathrm{NL}}\left(g\left(\left|\nabla_{\mathrm{NL}} u\right|\right) \nabla_{\mathrm{NL}} u\right)+\alpha V u_{0} .
$$

We enhance textures by combining two denoising processes. Since nonlocal methods have been proven to often outperform local methods in image denoising, we want as well to make the comparison in our context. The benefit of using a nonlocal equation to reconstruct a noisy pixel $x$ is that all its similar pixels in the image become involved in the reconstruction and not only its closest neighbors. To evaluate the similarity between pixels $x$ and $y$, one must define weights $w(x, y) \in[0,1]$ :

$$
\begin{aligned}
& d(x, y)=\quad e^{\frac{|p(x)-p(y)|^{2}}{4 \tau^{2}}} \\
& w(x, y)=\frac{1}{c(x)} e^{-\frac{|p(x)-p(y)|^{2}}{2 \tau^{2}}},
\end{aligned}
$$

where $p(x)$ is a patch of pixels around the pixel $x$ (square window of neighbors pixels), $\tau$ is a filtering parameter which controls the decay of the weights, and $c(x)=\int_{\Omega} w(x, y) d y$ is a normalizing factor. Other distances can be used as mentioned in [16].

In order to reduce computational time [15], we do not choose $\Omega$ to be the integration domain of the above integral equations, even if theoretically, it would have been better. In practice, we restrain the search of patches to a square search window of size $(2 s+1) \times(2 s+1)$. The patches $p(x)$ are of size $(2 p+1) \times(2 p+1)$ and usually $p \ll s$. A trade-off between $s$ and $p$ must be found.

\section{RESUlts}

In order to evaluate the performance of the different methods, we performed experiments on 10 textures adopted from the Kylberg dataset [17]. Original images are $576 \times 576$ grayscale textures, but to reduce the computational time, we cropped them and processed only $128 \times 128$ samples. To each texture, we added white Gaussian noise of standard deviation $\sigma=0.05,0.075,0.1$. Then we processed the images by means of different algorithms while optimizing the involved parameters ${ }^{1}$. Our goal is not to develop automatic methods to set the parameters but, instead, to find parameters that should achieve the best reconstruction. Therefore, all parameters were

\footnotetext{
${ }^{1}$ For the implementation, we used Matlab with the help of Numerical Tour toolboxes [18] and codes obtained from respective references.
}

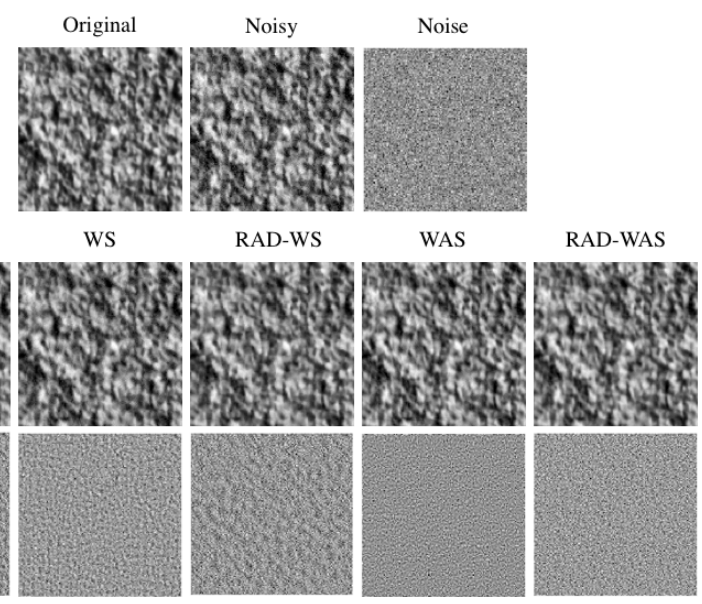

Fig. 1. The effect of adding a potential in local diffusion equation. Noisy wall texture with $\sigma=0.05$. Top row: original, noisy, and noise images. Middle row: RAD, WS, RAD-WS, WAS, RAD-WAS denoised images. Bottom row: corresponding residue images.

chosen to reach the best possible performance. As a criterion to compare noisy and reconstructed images, we used PSNR.

First simulations were performed on local algorithms. Independently of the type of texture, best performance was achieved by diffusion with potential RAD-WAS. Also, RADWS outperformed anisotropic diffusion. The results highlight the role played by the potential in texture enhancement. In particular, wave atoms are more suitable than wavelets to enhancing oscillatory and oriented textures [19]. Figure 1 illustrates visually the advantage afforded of adding a potential to diffusion process. Residue images correspond to the difference between reconstructed and input noisy images, and show the noise and details that have been removed by the applied process. Diffusion with potential results show residue that is less correlated with original texture. RAD-WAS and RADWS mostly outperformed WAS and WS filters. The diffusion (RAD) and the potential filters combine their strengths to produce a better reconstruction. Note in figure 1 the advantage of RAD-WAS, by observing that the residue image is nearly similar to that of noise, compared with the other methods, even with WAS.

When we consider each texture separately, we observe that the quality of the denoising depends on the type of texture. Figure 2 depicts RAD results of every texture sample on the $\mathrm{x}$-axis (in $\mathrm{dB}$ ), and the increase in PSNR due to incorporation of WAS potential on the y-axis ( $\triangle$ PSNR $=$ PSNR $_{\text {RAD-WAS }}-$ PSNR $_{\text {RAD }}$ ). Textures can be classified into 3 groups, according to the quality of their reconstruction by RAD (values on the x-axis): (a) low, (b) average, and (c) high denoising quality. In fact, textures belonging to the same group may share common properties. From group (a) to (c), the scale of characteristic features and the proportion of flat regions in textures increases. Textures with small details whose scale is close to noise scale are hardly denoised. The contribution of adding a WAS potential in order to improve RAD results 
differs also according to the texture, as it can be seen on the y-axis of figure 2. Best contributions appear in samples of group (a) and (b) (paille and wall texture images with a maximal improvement of $0.7 \mathrm{~dB}$ ) that contain oscillatory and oriented textured patches. Besides, as well as the quality of reconstruction, improvements due to the addition of a potential decrease with the level of noise.

Nonlocal algorithms were also tested. We used the NLmeans implementation of [18]. To reduce computational time, we extended the code and used a blockwise implementation as proposed in [20]. Instead of computing NLM averaging for all pixels in the search window, blockwise implementation performs weighted averaging of blocks (or patches) of pixels centered in every $q$ pixels. This simplification speeds up computations, but reduces denoising quality. On the contrary, for several textures, classical implementation enables to achieve higher performance than local methods. The influence of a potential is hardly observed with blockwise implementation. However, with classical implementation, PSNR improvements by $\sim 0.1 \mathrm{~dB}$ show the advantage of adding a potential, especially a wave atom potential. Improvements are more significative for certain textures, for oscillatory ones and textures with small scale features. The impact of the potential on reconstruction seems to be closely linked to particular denoising capabilities of the transform used in the potential. In fact, wave atom transform is more suitable for oscillatory patterns as shown in [19]. Figure 3 shows visually the benefit of combining a potential with nonlocal diffusion process. NLAD-WAS residue images are less correlated with original textures than NLMs.

PSNR results have hardly improved (improvements by $\sim 0.01$ $\mathrm{dB}$ ) by the introduction of a diffusivity in the nonlocal equation (equation (14)). We conclude that the analogy between local and nonlocal schemes is not so simple. Nonlocal diffusivity can be interpreted as adaptive weights but its efficacy in image denoising is not qualitatively justified. In order to extrapolate concepts introduced by Perona and Malik [2] to nonlocal denoising, adaptive weights models as proposed in [21] would be more relevant. However, we did not fully explore NLAD model mainly because of the difficulty to evaluate optimal parameters. In fact, the correlation between parameters $\tau$ and $k$ is not clear. High computational times do not facilitate the task of understanding this correlation.

\section{CONCLUSiON}

Diffusion with potential processes achieve texture enhancement. Both local and nonlocal approaches show advantages of adding a suitable potential to the diffusion equation. The diffusion with potential equation combines advantages characteristic of the two filters: the anisotropic diffusion filter allows to perform piecewise smoothing; the potential allows to reincorporate in the reconstructed image the loss of details caused by smoothing. The impact of the potential depends on the type of texture. Wave atom shrinkage potential is more suitable for oscillatory and small scale patterns.

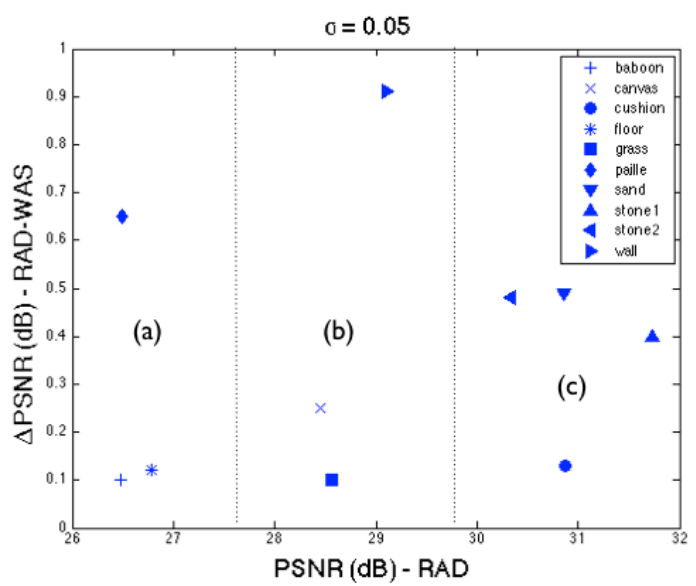

(a)
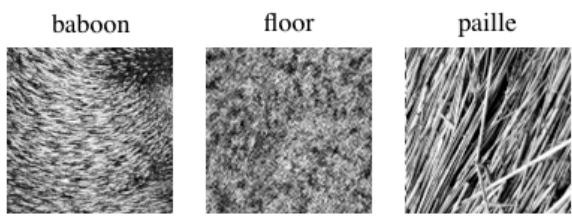

grass

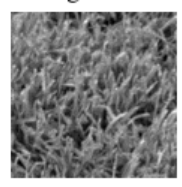

canvas

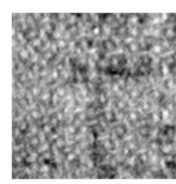

wall

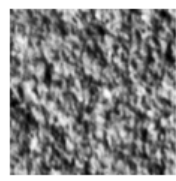

cushion
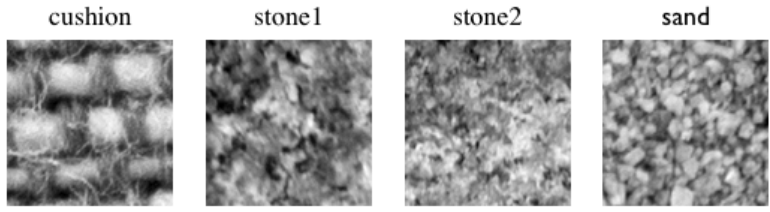

Fig. 2. Classification of results. Top: on the x-axis, PSNR results by RAD; on the y-axis: $\triangle$ PSNR $=$ PSNR $_{\text {RAD-WAS }}-$ PSNR $_{\text {RAD }}$ : the increase of PSNR by adding WAS potential to RAD. Quality of the reconstruction of textures: (a) low, (b) medium, (c) high (see bottom images). Level of noise is $\sigma=0.05$.

Since texture enhancement depends on types of texture, a nonlocal diffusion with multiple potentials may be envisaged. By using statistical criteria in order to classify textured patches in the input image, one may incorporate an appropriate potential to every patch. Nonlocal methods should facilitate classification and adaptive denoising of patches. Also, one should enlarge the list of useful potentials and classify them according to their impact on textures. Besides, the optimization of parameters must be improved. In particular, nonlocal framework cannot be totally explored, unless computational times have been strongly reduced with the same quality of denoising. Reducing computational time will allow to perform more relevant simulations and may help to deduce approximative estimators of parameters. The estimation should depend on the noise level and some intrinsic properties of textures. 


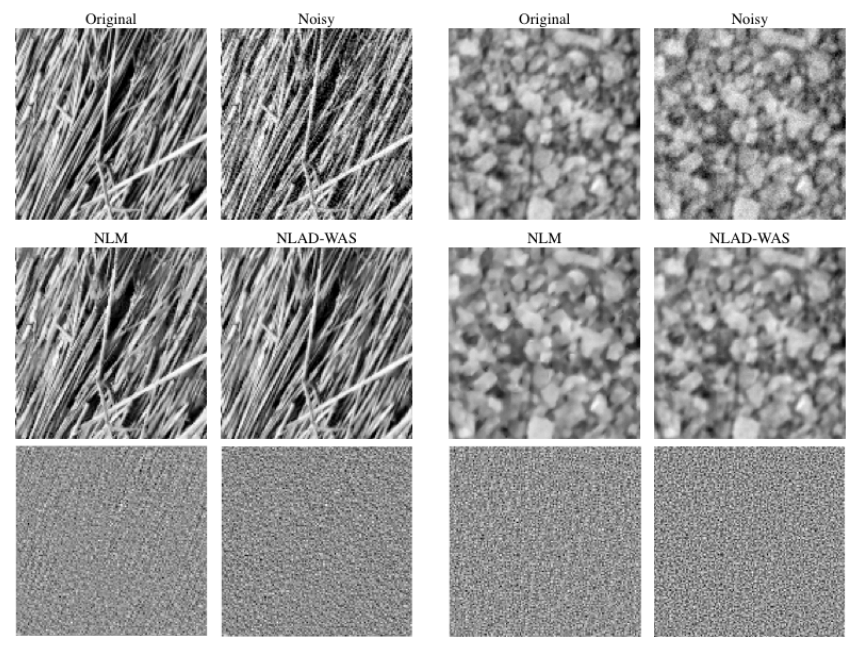

Fig. 3. Benefit of adding a potential to nonlocal diffusion equation. Paille (left) and sand (right) textures respectively for $\sigma=0.1$ and $\sigma=0.05$. From top to bottom: original and noisy images; NLM and NLAD-WAS denoised images; corresponding residue images.

\section{ACKNOWLEDGMENT}

The authors would like to thank Vadim Ratner for his help and advice. This research was supported by the Ollendorff Minerva Center.

\section{REFERENCES}

[1] J. Weickert, "A review of nonlinear diffusion filtering," in Scale-Space Theory in Computer Vision, vol. 1252, pp. 1-28. Springer, 1997.

[2] P. Perona and J. Malik, "Scale-space and edge detection using anisotropic diffusion," IEEE Trans. Pattern Analysis and Machine Intelligence, vol. 12, no. 7, pp. 629-639, 1990.

[3] L.I. Rudin, S. Osher, and E. Fatemi, "Nonlinear total variation based noise removal algorithms," Physica D: Nonlinear Phenomena, vol. 60, no. 14 , pp. 259-268, 1992.

[4] R. Deriche and O. Faugeras, "Les équations aux dérivées partielles en traitement des images et vision par ordinateur," Traitement du signal, vol. 13, no. 6, pp. 551-577, 1996.

[5] G. Gilboa, N. Sochen, and Y.Y. Zeevi, "Forward-and-backward diffusion processes for adaptive image enhancement and denoising," IEEE Trans. Image Proc., vol. 11, no. 7, pp. 689-703, 2002.

[6] G. Gilboa, N. Sochen, and Y.Y. Zeevi, "Image enhancement and denoising by complex diffusion processes," IEEE Trans. Pattern Analysis and Machine Intelligence, vol. 26, no. 8, pp. 1020-1036, 2004.

[7] L.A. Vese and S.J. Osher, "Modeling textures with total variation minimization and oscillating patterns in image processing," Journal of Scientific Computing, vol. 19, no. 1-3, pp. 553-572, 2003.

[8] O. Honigman and Y.Y. Zeevi, "Enhancement of textured images using complex diffusion incorporating schrodinger's potential," in ICASSP 2006 Proc. IEEE, vol. 2, pp. II-633-II-636, 2006.

[9] G. Plonka and J. Ma, "Nonlinear regularized reaction-diffusion filters for denoising of images with textures," IEEE Trans. Image Proc., vol. 17, no. 8, pp. 1283-1294, 2008.

[10] G. Gilboa and S. Osher, "Nonlocal operators with applications to image processing," Multiscale Modeling and Simulation, vol. 7, no. 3, pp. 1005$1028,2008$.

[11] C. Tsiotsios and M. Petrou, "On the choice of the parameters for anisotropic diffusion in image processing," Pattern Recognition, vol. 46, no. 5, pp. 1369 - 1381, 2013.

[12] F. Catté, P.-L. Lions, J.-M. Morel, and T. Coll, "Image selective smoothing and edge detection by nonlinear diffusion," SIAM Journal on Numerical Analysis, vol. 29, no. 1, pp. 182-193, 1992.

[13] V. Ratner and Y.Y. Zeevi, "Stable denoising-enhancement of images by telegraph-diffusion operators," in ICIP 2013 Proc. IEEE, 2013.
[14] D. Zhou and B. Schölkopf, "Regularization on discrete spaces," in Pattern Recognition, vol. 3663, pp. 361-368. Springer, 2005.

[15] A. Buades, B. Coll, and J.-M. Morel, "A non-local algorithm for image denoising," in CVPR 2005. IEEE, vol. 2, pp. 60-65, 2005.

[16] G. Gilboa and S. Osher, "Nonlocal linear image regularization and supervised segmentation," Multiscale Modeling and Simulation, vol. 6, no. 2, pp. 595-630, 2007.

[17] Gustaf K., "The kylberg texture dataset v. 1.0," Tech. Rep. 35, Centre for Image Analysis, Uppsala, Sweden, 2011.

[18] G. Peyré, "The numerical tours of signal processing - advanced computational signal and image processing," IEEE Computing in Science and Engineering, vol. 13, no. 4, pp. 94-97, 2011.

[19] L. Demanet and L. Ying, "Wave atoms and sparsity of oscillatory patterns," Applied and Computational Harmonic Analysis, vol. 23, no. 3 , pp. 368-387, 2007.

[20] P. Coupé, P. Yger, S. Prima, P. Hellier, C. Kervrann, and C. Barillot, "An optimized blockwise nonlocal means denoising filter for 3-d magnetic resonance images," IEEE Trans. Medical Imaging, vol. 27, no. 4, pp. $425-441,2008$.

[21] M. Mahmoudi and G. Sapiro, "Fast image and video denoising via nonlocal means of similar neighborhoods," IEEE Signal Processing Letters, vol. 12, no. 12, pp. 839-842, 2005. 